\title{
Diffusion and Home Range Parameters from Rodent Population Measurements in Panama
}

\author{
L. Giuggioli ${ }^{\text {a }}$, G. Abramson ${ }^{\mathrm{a}, \mathrm{b}}, \mathrm{V}$. M. Kenkre ${ }^{\mathrm{a}}$ \\ ${ }^{a}$ Consortium of the Americas for Interdisciplinary Science, University of New \\ Mexico, Albuquerque, New Mexico 87131, USA. \\ ${ }^{\mathrm{b}}$ Centro Atómico Bariloche, CONICET and Instituto Balseiro, 8400 San Carlos de \\ Bariloche, Río Negro, Argentina.
}

G. Suzán, E. Marcé, T. L. Yates

Department of Biology, University of New Mexico, Albuquerque, New Mexico 87131, USA.

\begin{abstract}
Simple random walk considerations are used to interpret rodent population data collected in Hantavirus-related investigations in Panama regarding the short-tailed cane mouse, Zygodontomys brevicauda. The diffusion constant of mice is evaluated to be of the order of (and larger than) 200 meters squared per day. The investigation also shows that the rodent mean square displacement saturates in time, indicating the existence of a spatial scale which could, in principle, be the home range of the rodents. This home range is concluded to be of the order of 70 meters. Theoretical analysis is provided for interpreting animal movement data in terms of an interplay of the home ranges, the diffusion constant, and the size of the grid used to monitor the movement. The study gives impetus to a substantial modification of existing theory of the spread of the Hantavirus epidemic which has been based on simple diffusive motion of the rodents, and additionally emphasizes the importance for developing more accurate techniques for the measurement of rodent movement.
\end{abstract}

Key words: Hantavirus, Zygodontomys, animal diffusion, home range, random walk

Email addresses: giuggiol@unm.edu (L. Giuggioli), abramson@cab.cnea.gov.ar (G. Abramson), kenkre@unm.edu (V. M. Kenkre), gsuzan@unm.edu (G. Suzán), emarce@unm.edu (E. Marcé), tyates@unm.edu (T. L. Yates).

Preprint submitted to Bulletin of Mathematical Biology 14 November 2018 


\section{Introduction}

The Hantavirus epidemic is of great concern to human health in many regions of the world (Yates et al., 2002; Mills et al., 1999; Parmenter et al., 1999). The discovery of Hantavirus in the New World took place after an outbreak of a severe disease in the region of the Four Corners in the North American Southwest, in 1993. The agent, Hantavirus Sin Nombre, is carried mainly by the extremely common deer mouse, Peromyscus maniculatus (Nichol et al., 1993; Childs et al., 1994). Since the discovery, an enormous effort has been devoted to understanding the ecology and epidemiology of the virus-mouse association, with the ultimate goal being prediction of human risk (Mills et al., 1999; Parmenter et al., 1998). Numerous species of the virus are known in the Americas, each one of them almost exclusively associated with a single rodent reservoir (Schmaliohn et al., 1997). Human disease caused by these pathogens can range from mild to very severe, with a mortality rate in some cases approaching $50 \%$.

A theory for the spread of the Hantavirus was constructed a few years ago by two of the present authors (Abramson and Kenkre, 2002) and shown to lead naturally to spatiotemporal patterns such as the observed refugia (Yates et al., 2002) and the sporadic disappearance and appearance of the epidemic (Mills et al., 1999; Parmenter et al., 1999). That theory will be referred to in the rest of the paper as AK. Seasonal, as well as extraordinary variations in demographic and environmental conditions are included in the AK model through spatiotemporal dependence of several parameters, such as the carrying capacity. The emergence of traveling waves of infection (Abramson et al., 2003), the investigation of fluctuations (Aguirre et al., 2002), external changes in environmental effects (Ballard et al., 2004), and other features (Kenkre, 2003; Abramson, 2003; Kenkre, 2004; Buceta et al., 2004) have also been studied. There are additional factors, not yet analyzed theoretically, which may be of importance in the dynamics of the virus. For example, human activity such as changes in agricultural practice may alter habitats and drive the rodent population into new habitats not previously occupied by them. While many theoretical issues regarding the AK development have been, and are being, explored quite intensely, the major problem of obtaining the values of the parameters inherent in the theory has remained neglected as a result of paucity of available data. Needless to say, the solution of this problem is crucial to the quantitative description of the spread of the epidemic. The purpose of the present paper is such extraction of the essential parameters necessary in the description of the spread of the Hantavirus.

The AK model (Abramson and Kenkre, 2002; Abramson et al., 2003) is based on a fundamental set of biological features that characterize the transmission of Hantavirus among rodent populations, and involves five parameters: the birth 
rate $b$ of the rodents (mice), their death rate $c$, the environmental parameter $K$, the contagion rate $a$, and the diffusion coefficient $D$. In terms of these, the mice populations $M_{s}$ (susceptible) and $M_{i}$ (infected) obey

$$
\begin{aligned}
& \frac{\partial M_{s}}{\partial t}=b\left(M_{s}+M_{i}\right)-c M_{s}-\frac{M_{s}\left(M_{s}+M_{i}\right)}{K(x, t)}-a M_{s} M_{i}+D \nabla^{2} M_{s}, \\
& \frac{\partial M_{i}}{\partial t}=-c M_{i}-\frac{M_{i}\left(M_{s}+M_{i}\right)}{K(x, t)}+a M_{s} M_{i}+D \nabla^{2} M_{i} .
\end{aligned}
$$

All the parameters except $K$ are considered to be independent of time $t$ and space $x$.

The measurement of the birth and death rates $b, c$ presents no special challenge. The environmental parameter $K$ is sometimes measured from food and vegetation measurements, and also often obtained from aerial photographs of the landscape. Its variation in time and space can be well characterized although its absolute values are difficult to obtain. The encounter infection rate $a$ is notoriously hard to measure from observations of individual mouse-mouse

Table 1

Summary of the mark-recapture data of $Z$. brevicauda. A total of 846 captures, corresponding to 411 different animals, were obtained. Of them, 188 were captured at least twice, and at most 10 times. The probability of recapture depends on sex and age group, as shown. It suggests an increase of the probability of recapture with age, independently supported by the probability of recapture as a function of weight (not shown). J: juveniles, SA: sub-adults, A: adults, F: females, M: males.

\begin{tabular}{lrrrr}
\hline \hline & J & SA & A & Total \\
\hline Captured only once: & 7 & 13 & 98 & 118 \\
F & 8 & 18 & 79 & 105 \\
M & 15 & 31 & 177 & 223 \\
Total & & & & \\
\hline Recaptured at least once: & 2 & 2 & 71 & 75 \\
F & 0 & 16 & 97 & 113 \\
M & 2 & 18 & 168 & 188 \\
Total & & & & \\
\hline Probability of recapture: & 0.22 & 0.13 & 0.42 & \\
F & 0.00 & 0.47 & 0.55 & \\
M & 0.13 & 0.37 & 0.49 & \\
Total & & & & \\
\hline \hline
\end{tabular}


interactions (Botten et al., 2002). At this stage of observational technique, we must assume that it is a floating parameter.

The last of the five parameters, the diffusion constant $D$, is crucial to the AK description since the assumed mechanism for the spread of the epidemic is the diffusion (movement) of infected mice over the terrain followed by the transmission of infection to susceptible mice. In principle, it appears straightforward to measure $D$ from records of mice movement in a mark-recapture experiment, as was done by Ovaskainen (2004) in a recent study on the dispersal of butterflies in a heterogeneous habitat. The investigation reported in the present paper began as an attempt to extract $D$ directly in that manner. We will see that examination of the data has indeed allowed us to obtain values of $D$ but also led us to a number of important conclusions about the transmission of Hantavirus among rodent populations, and has suggested substantial changes to be introduced into the theory of the spread of Hantavirus.

\section{The data set: Zygodontomys brevicauda in Panama}

At the beginning of 2000, human cases (more than 20 in the first cluster) of Hantavirus Pulmonary Syndrome (HPS) were recognized from the Azuero Peninsula, Panama. Following the outbreak, it was discovered that the pigmy rice rat Oligoryzomys fulvescens and the short-tailed cane mouse Zygodontomys brevicauda harbored two novel hantaviruses, Choclo virus (responsible for the HPS cases), and Calabazo virus (not known to cause human disease) respectively (Vincent et al., 2000). The data set we have selected in this study was obtained as the result of a mark-recapture observation performed in the Azuero Peninsula, in Tonosí (Los Santos), Panama, from June 27 to November 20, 2003. The observation corresponds to the rainy season in the region, and following several years of relative draught. During these years, clinical cases of HPS were rare (after being more frequent in 1999-2001) and rodent densities were probably relatively low.

Measurements were made on several species of rodents, of which we choose for the purposes of the present paper $Z$. brevicauda, host of Hantavirus Calabazo. This choice was made because $Z$. brevicauda was the most abundant species in the field study. A summary of the characteristics of the populations is presented in Table 1. The data relevant to the analysis of the movement consist of the position and time of capture of those mice that are captured at least twice, thus allowing for the calculation of their displacements from one location to another. The measurements were made with a square array of $7 \times 7$ Sherman traps, separated $10 \mathrm{~m}$ each. Each measuring session lasted 3 days, with a time of recurrence to the same site of about one month. The number of trapping grids used in the study was 24, in 4 different sites. Each grid was set up across 


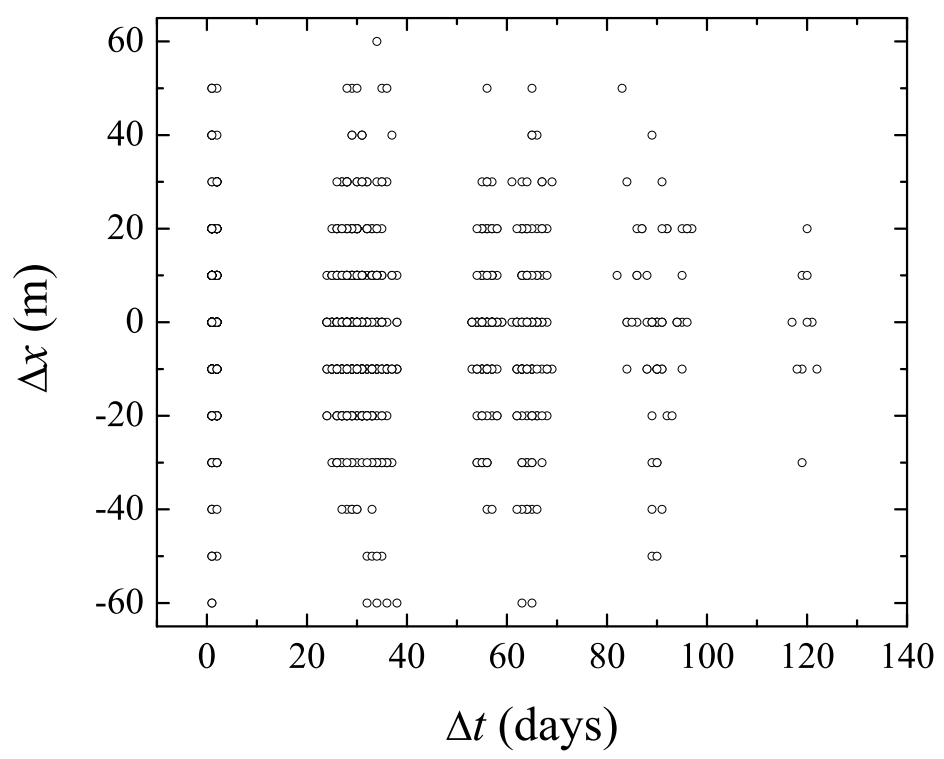

Fig. 1. Displacements of the recaptured mice, projected along the $x$ direction which is the direction into and out of the forest relative to the pastures in the landscape. Each point corresponds to a displacement of one of the 411 mice that were captured more than once. Some mice were captured more than twice, thus contributing with more than one point to this set.

the edge between forest and pastures (Suzán et al., 2004). For our analysis given below, all grids have been rotated such that the edge runs along the $y$ direction.

\section{The movement of $Z$. brevicauda}

A few animals were captured a sufficient number of times (about 10), during a period of months, as to allow us to form a useful picture of the mouse walks. Unfortunately, there are only five of these mice. The limited number makes it impossible to carry out any statistical analysis of the properties of the walks.

Despite this drawback, we were able to proceed with the analysis because the data set contains hundreds of recapture events, corresponding to different mice, each one providing us with a displacement at a certain time scale. These time scales are 1 and 2 days (if the recapture occurs during the same session, that lasts three days); about 1 month (if the recapture occurs at the next session); and about 2 months (at the second next session), 3 months, and 4 months, respectively. At progressively longer time scales there are, certainly, less recapture events; but there are a sufficient number of them for sensible results to be obtained up to the 3 -month scale. The data are shown in Fig. 1, 
for the $x$-component of the 2-dimensional displacements of the mice. Each point in the graph represents a displacement $\Delta x$ taking place during an interval $\Delta t$. Each displacement corresponds to the movement of a single mouse, but different displacements may or may not correspond to the same animal. We consider these displacements as our elementary events. Furthermore, we specifically assume them to be statistically independent. There are repeated events in the set, namely displacements with the same $\Delta x$ and $\Delta t$, a fact not represented in Fig. 1. In the plot, it can be observed that, besides the 1- and 2-day intervals, the data are scattered in "clouds" around 1, 2, 3 and 4 months. The reason for this is twofold. First, each session consists of 3 consecutive days of capture; therefore, the interval between two recaptures in different sessions is not a constant number of days. Second, variations due to the logistics of field work result in the time between sessions not being precisely 30 days.

On each time scale, the set of available displacements is taken to represent a statistical ensemble, i.e., a population of ideal mice with certain statistical properties. Also, the displacements measured on each time scale correspond to a progressively coarser graining of the actual mice walks, containing an indeterminate (but presumably large) number of steps already on the 1-day scale.

Using available data on each time scale, we construct mouse walks by randomly shuffling the displacements. These walks represent instances of possible walks, on that time scale, with exactly the same statistical properties as a hypothetical "representative mouse". We construct various such walks and perform ensemble averages to obtain the mean square displacement as a function of time. For example, on the time scale of 1 day, 20 instances of the walk produce the result shown in Fig. 2. Diffusive behavior is inferred from the clearly linear rise of the mean square displacement. On longer time scales the same analysis produces smaller diffusion coefficients. However, application of the method is not reliable on these longer scales because it does not permit a correct evaluation of errors.

The above considerations clarify the underlying diffusive nature of mice motion. A direct analysis of the probability distribution function $P(\Delta x)$ is possible, also on each time scale. $P(\Delta x)$ is a bell-shaped distribution, with a well-defined variance that can be used to characterize the evolution of the mean square displacement of the representative mouse as a function of time. The result of this analysis is in Fig. 3, where we show the mean square displacements in both the $x$ and the $y$ directions. Unlike in a simple diffusive process, the observed mean square displacement is observed to saturate to a value near $400 \mathrm{~m}^{2}$. The saturation value is different for $\left\langle\Delta x^{2}\right\rangle$ and for $\left\langle\Delta y^{2}\right\rangle$, indicating an anisotropy in the system. We have already mentioned above that each trapping grid contains an edge between forest and pastures, mainly along the $y$ direction. This is surely responsible for the anisotropy. 


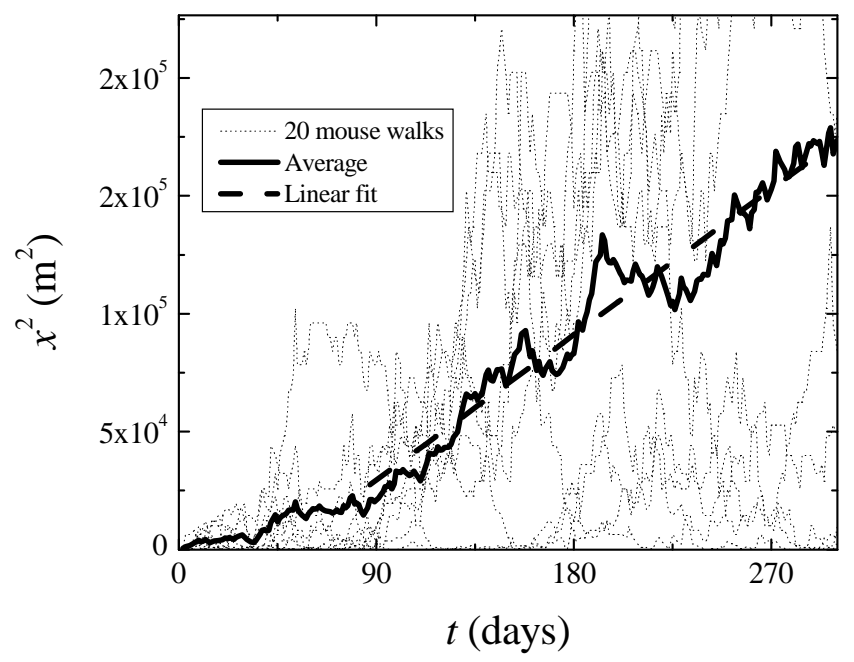

Fig. 2. Square of the displacement as a function of time, on the time scale of 1 day, for 20 artificial "mouse walks" based on the recorded displacements. The heavy line is an ensemble average.

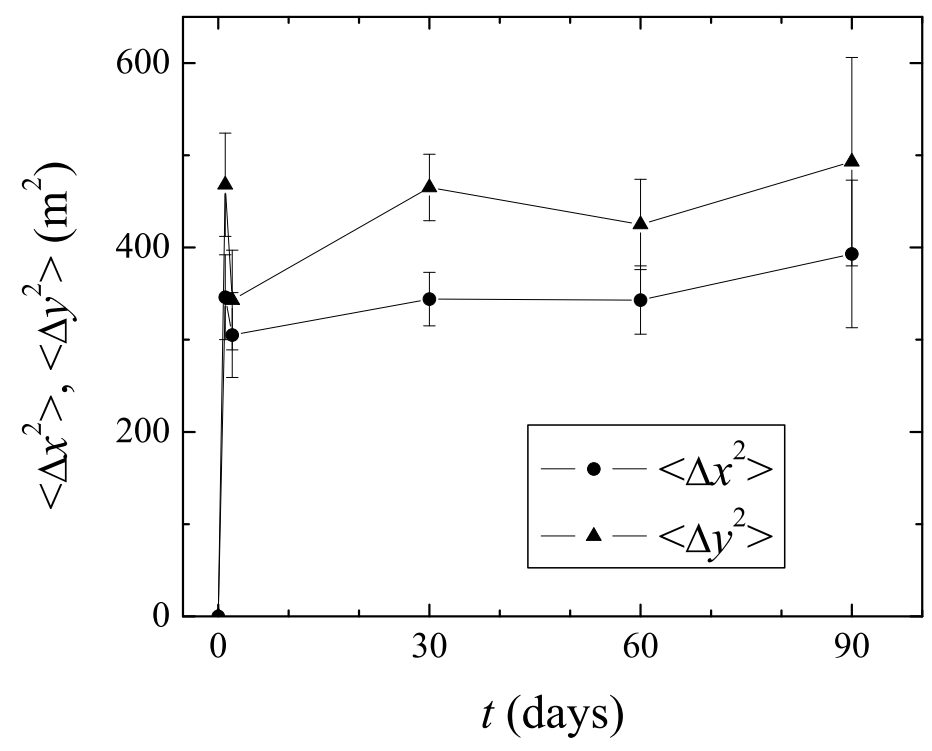

Fig. 3. Mean square displacement as a function of time, using the different time scales available from the data of Fig. 1. The two curves correspond to the two directions in space. 


\section{Movement in Confined Spaces: Rodent Home Ranges and Grid Sizes}

The saturation of the mean square displacement, clear from the observations discussed above, implies the existence of a spatial scale in the rodent system. One obvious possibility is that the saturation is a manifestation of the confined motion of the rodents, i.e., the existence of home ranges (Burt, 1943). In order to treat the problem quantitatively, we first present a simple calculation of the effect of confinement on the mean square displacement of a random walker.

\subsection{Effect of Confinement on $\left\langle x^{2}\right\rangle$}

There are several ways one might model the effect of confinement on the motion of rodents. One is to treat the motion as obeying, not a diffusion equation, but a Fokker-Planck equation in an attractive potential (Kuperman et al., 2004). A simpler way, which we adopt here, is to consider the motion as occurring via simple diffusion but confined to a box whose size represents the home range. Restricting the analysis to 1-dimension for simplicity, we solve the diffusion equation for the probability per unit length $P(x, t)$ of finding the mouse at position $x$ and time $t$ inside a bounded domain of length $L$. Taking into account the symmetry of the problem, the general solution can be written as $P(x, t)=A_{0}+\sum A_{\lambda} \cos [\lambda x] e^{-\lambda^{2} D t}$. By imposing the condition that a mouse cannot escape from the home range (zero flux boundary condition), the allowed $\lambda$ coefficients can be calculated and the solution written as

$$
P(x, t)=\frac{1}{L}+\frac{2}{L} \sum_{n=1}^{+\infty} A_{n} \cos \left[\frac{2 n \pi x}{L}\right] e^{-\frac{(2 n)^{2} \pi^{2} D t}{L^{2}}},
$$

wherein

$$
A_{n}=\frac{2}{L} \int_{-L / 2}^{L / 2} d x \cos \left[\frac{2 n \pi x}{L}\right] P(x, 0) .
$$

With the initial condition that the mouse is within a length $\alpha$ centered around the origin of the box, specifically

$$
P(x, 0)=\left\{\begin{array}{ll}
1 / \alpha & |x| \leq \alpha / 2 \\
0 & |x|>\alpha / 2
\end{array},\right.
$$

the evolution at all times can be written as

$$
P(x, t)=\frac{1}{L}+\frac{2}{\alpha \pi} \sum_{n=1}^{+\infty} \frac{\sin \left(\frac{n \pi \alpha}{L}\right) \cos \left(\frac{2 n \pi x}{L}\right)}{n} e^{-\frac{(2 n)^{2} \pi^{2} D t}{L^{2}}} .
$$


The initial condition (5) reduces to a $P(x, 0)=\delta(x)$ in the limit $\alpha \rightarrow 0$. The calculation of the mean square displacement $\left\langle x^{2}\right\rangle=\int_{-L / 2}^{L / 2} d x x^{2} P(x, t)$ gives

$$
\left\langle x^{2}\right\rangle=\frac{L^{2}}{12}\left\{1-\frac{L}{\alpha \pi^{3}} \sum_{n=1}^{+\infty} \frac{(-1)^{n+1} \sin \left(\frac{n \pi \alpha}{L}\right)}{n^{3}} e^{-\frac{(2 n)^{2} \pi^{2} D t}{L^{2}}}\right\} .
$$

We see that $\left\langle x^{2}\right\rangle \simeq \alpha^{2} / 12+2 D t$ for $t \rightarrow 0$. We also see that $\left\langle x^{2}\right\rangle$ saturates to $L^{2} / 12$ for $t \rightarrow+\infty$. If $P(x, 0)=\delta(x)$, the mouse will move initially as if no home range existed. However, its diffusive motion will be limited in extent by the presence of the home range and $\left\langle x^{2}\right\rangle$ will eventually saturate. The 1-dimensional calculation captures the essential features. The 2-dimensional extension, appropriate to mouse movement on the terrain, is straightforward to obtain because $P(x, y, t)$ is given by the product of two functions of the form (6), one for the $x$-plane with $D_{x}$ and $L_{x}$ and the other for the $y$-plane with $D_{y}$ and $L_{y}$. Allowing for the fact that differences in terrain could be reflected in the differences in $D_{x}$ and $D_{y}$ (given that the traps are laid out systematically relative to 'edges' in the landscape between forests and pastures), we give a usable expression for the average mean square displacement in 2-dimensions which can be used directly for the interpretation of the observations.

$$
\begin{aligned}
\left\langle x^{2}\right\rangle & +\left\langle y^{2}\right\rangle=\frac{L_{x}^{2}+L_{y}^{2}}{12}\left[1-\frac{1}{\alpha \pi^{3}} \sum_{n=1}^{+\infty} \frac{(-1)^{n+1}}{n^{3}} \times\right. \\
& \left.\times\left\{L_{x} \sin \left(\frac{n \pi \alpha}{L_{x}}\right) e^{-\frac{(2 n)^{2} \pi^{2} D_{x} t}{L_{x}^{2}}}+L_{y} \sin \left(\frac{n \pi \alpha}{L_{y}}\right) e^{-\frac{(2 n)^{2} \pi^{2} D_{y} t}{L_{y}^{2}}}\right\}\right] .
\end{aligned}
$$

Application of this analysis to the mouse data is straightforward. The shorttime part of the mean square displacement gives the diffusion constant, averaged over the two directions, to be $200 \pm 50 \mathrm{~m}^{2} / \mathrm{d}$. The saturation value appears to imply that the home range $L$ equals about $70 \mathrm{~m}$. While these initial considerations suggest that the Panama data allow us to confirm the existence of rodent home ranges, as well as to measure their extent, careful observation introduces a note of caution: we notice that the derived value of the home range is of the order of the size of the measurement grid $G=60 \mathrm{~m}$. Could sampling from a limited domain in space lead to saturation and mislead one into drawing incorrect conclusions about the home range? To answer this question we carry out the following analysis.

\subsection{Effect of Limited Spatial Observations on $\left\langle x^{2}\right\rangle$}

Consider the motion of a random walker in unbounded space (no home ranges) but let the mean square displacement be calculated from observations in a 
limited part of space of size $G$ placed symmetrically around the origin for simplicity. We obviously have

$$
\left\langle x^{2}\right\rangle=\frac{\int_{-G / 2}^{G / 2} d x x^{2} P(x, t)}{\int_{-G / 2}^{G / 2} d x P(x, t)}
$$

where the probability $P(x, t)$ is given by the propagator of the diffusion equation in unbounded space:

$$
P(x, t)=\frac{e^{-\frac{x^{2}}{4 D t}}}{\sqrt{4 \pi D t}}
$$

Substitution gives

$$
\left\langle x^{2}\right\rangle=2 D t\left[1-\frac{G e^{-\frac{G^{2}}{16 D t}}}{\sqrt{4 \pi D t} \operatorname{erf}\left(\frac{G}{4 \sqrt{D t}}\right)}\right] .
$$

At short times $\left\langle x^{2}\right\rangle \simeq 2 D t$ while at long times it saturates to the value $G^{2} / 12$. The 2-dimensional result is trivially obtained as a generalization of (11).

Note that the behavior of a system without home ranges but with a finite window of observation is qualitatively different from that of a system with home ranges and an infinitely large window. The mean square displacement in the former (Eq. (11)) has quite a different time dependence from the latter (Eq. (7)), since the error function expressions differ considerably from exponentials. It is easy to see that in both cases the mean square displacement starts out at short times as $2 D t$ and saturates to a constant at long times. The saturation is to $G^{2} / 12$ in the first case and to $L^{2} / 12$ in the second. There is thus potential for confusion. One could mistakenly interpret what is actually the measurement grid size $G$ to be the rodent home range $L$.

Combination of the two elements discussed above, the home range effect and the grid size effect, is straightforward to analyze. Consider the situation in which a mouse is moving randomly inside a home range of width $L$ but is observed only inside a region of width $G$, both concentric for simplicity. If the grid is larger than the home range $(G>L)$, the evolution of the mean square displacement is exactly given by the previous result (7). In the other case, 


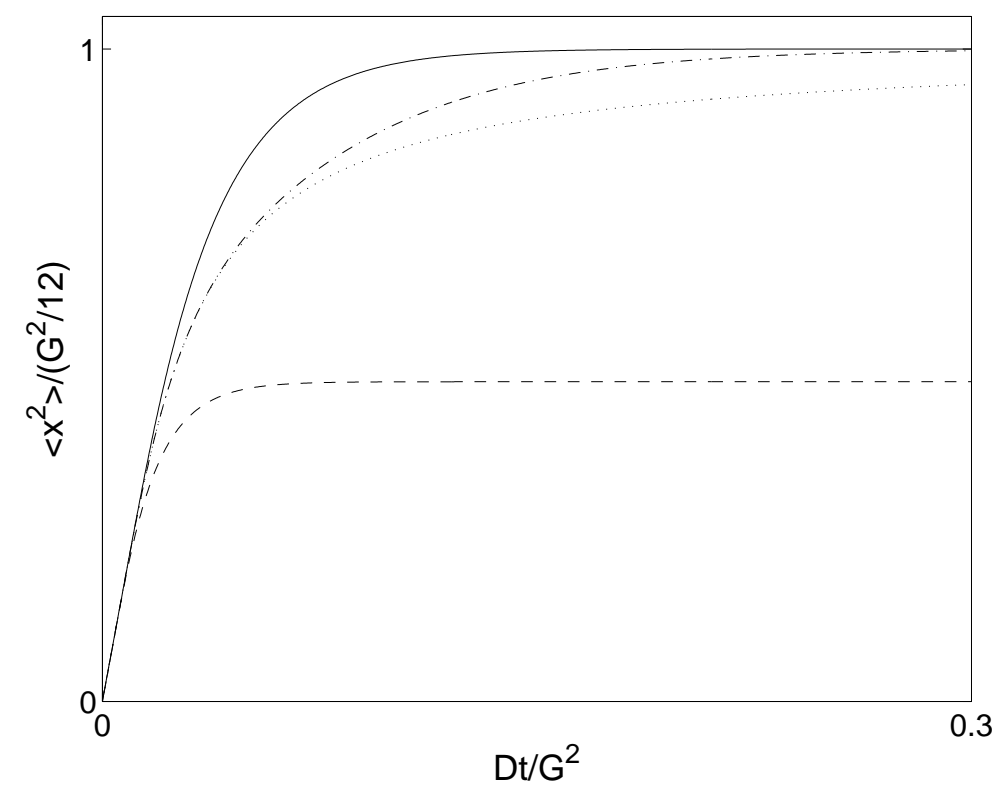

Fig. 4. Mean square displacement in units of $G^{2} / 12$ as a function of time in units of $G^{2} / D$ for different values of the ratio $G / L$, i.e., the ratio of the measuring grid divided by the width of the home range. The dashed, solid, dash-dotted and dotted lines correspond, respectively, to $G / L=1.43,1,0.7,0$. When $G>L,\left\langle x^{2}\right\rangle$ is seen to saturate to the lower value $L^{2} / 12$, whereas for all other $(G \leq L)$ cases, it saturates to $G^{2} / 12$. The time dependence differs qualitatively for the various cases. The difference might be discernible — in principle — with more refined measurements.

$(G<L)$, the evolution is

$$
\begin{aligned}
& \left\langle x^{2}\right\rangle=\frac{G^{2}}{12} \times \\
& \left\{\frac{\zeta+\frac{6 L}{\alpha \zeta^{2} \pi^{4}} \sum_{n=1}^{+\infty} \sin \left(\frac{n \pi \alpha}{L}\right)\left\{2 \pi \zeta \frac{\cos (n \pi \zeta)}{n^{3}}+\sin (n \pi \zeta)\left[\frac{\pi^{2}}{n^{2}} \zeta^{2}-\frac{2}{n^{4}}\right]\right\} e^{-\frac{(2 n) 2 \pi^{2} D t}{L^{2}}}}{\zeta+\frac{2 L}{\alpha \pi^{2}} \sum_{n=1}^{+\infty} \frac{\sin \left(\frac{n \pi \alpha}{L}\right) \sin (n \pi \zeta)}{n^{2}} e^{-\frac{(2 n)^{2} \pi^{2} D t}{L^{2}}}}\right\},
\end{aligned}
$$

wherein $\zeta=G / L$. Equation (12) reduces to Eq. (7) when $\zeta=1$. It can be shown that $\left\langle x^{2}\right\rangle$ goes as $\alpha^{2} / 12+2 D t$ for $t \rightarrow 0$ and saturates to $L^{2} / 12$ for $t \rightarrow+\infty$. In Fig. (4) we compare Eqs. (7), (11) and (12) for an initially localized condition $P(x, 0)=\delta(x)$. Despite the fact that all of the three curves (with $G<L$ ) show the same linear behavior at short times and reach the same saturation value, they differ considerably at intermediate times. A fourth curve (dashed, lowermost in the group) for which $G>L$ is also shown in the same graph.

An important step in the interpretation of saturation data is the verification of the sensitivity of the theoretical prediction to the initial position of the 


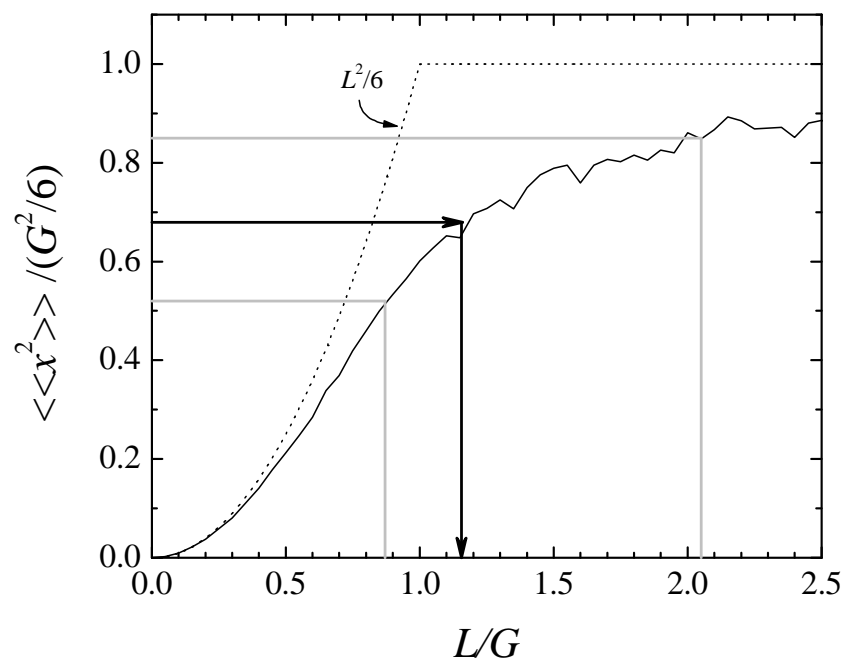

Fig. 5. Mean square displacement in units of $G^{2} / 6$ as a function of the ratio of the home range size to the grid size, $L / G$. The curve is the result of a simulation of 105 steps for each value of $L / G$. The arrow shows the average (in both directions $x$ and $y$ ) mean square displacement observed in the measurement, from the data shown in Fig. 3, and the inferred value of the home range $(L / G=1.16)$. The grey lines show the error bounds. The dotted lines show the analytic result derived in the Appendix, valid for home ranges concentric with the grid.

mice, as well as to the position of the home ranges with respect to the grid. In the Appendix we provide further details on the calculation of the mean square displacement, relevant to the dependence on the distribution of initial locations of the mice. To assess the combined effect, we have done a numerical experiment in which we measure simulated mice displacements. Each mouse is supposed to occupy uniformly its own home range, and all the home ranges are uniformly distributed in space. We limit the calculation of the mean square displacement to a window of size $G$, thus simulating the conditions of the measurement in the field. The result is shown in Fig. 5, where the normalized mean square displacement is plotted as a function of the normalized home range size $L / G$. The available data allow us to conclude that the home range of $Z$. brevicauda in Panama (averaged in the two directions) is $70 \mathrm{~m}$, with asymmetric error bounds from 50 to $120 \mathrm{~m}$.

\section{Discussion}

Extraction of quantitative information concerning the parameters in the Abramson-Kenkre theory (Abramson and Kenkre, 2002) of Hantavirus spread 
was the initial task undertaken in the present investigation. This was prompted by the success of that theory in reproducing qualitatively observed features such as the sporadic disappearance of infection during time periods of low carrying capacity, and the existence of refugia where infection persists and from where it propagates in the form of waves when conditions become favorable for such propagation. The key to the AK description of these processes was a transcritical bifurcation predicted by the theory and controlled by the carrying capacity. Crucial to quantitative application of the theory is the diffusion constant of the rodents, and the first aim of the present investigation was to extract this diffusion constant from movement observations collected for $Z y$ godontomys brevicauda in the peninsula de Azuero in Panama in a 5-month period in 2003. While logistic growth which is also part of the theory of ref (Abramson and Kenkre, 2002) is generally considered well established, both from field and laboratory studies (Murrav, 1993), equally strong justification for assuming diffusive transport for rodent movement is not available. The goal of extracting the diffusion constant has been met. The result particular to the species and location considered is $D=200 \pm 50 \mathrm{~m}^{2}$ per day.

During the process of this extraction, we encountered a spatial scale in the rodent system which could be representative of the home range of the mice. Preliminary investigations showed that the observed spatial scale could be reflecting the size of the measuring grid instead of a characteristic of the rodent system. Since accuracy of interpretation will be considerably increased by having multiple values of the grid size, our analysis underscores the importance for additional observations to be undertaken with grids of varying size. Motivated by this idea, we carried out further developments of the our theory which resulted in the simulation curve shown in Fig. 5 . The mean value of the home range for the two directions can be read off from the plot to be $L_{x}=60$ $\mathrm{m}$ and $L_{y}=90 \mathrm{~m}$.

Because our prescription for extracting the diffusion constant relies on the initial time evolution of the mean square displacement rather than its saturation value, we have been successful in obtaining a usable estimate of the diffusion constant. However, even that evaluation suffers from the fact that reconstruction of mouse walks from observations is difficult because multiple measurements for individual mice are extremely rare. Therefore, we suggest strongly that systematic measurements of rodent movement be undertaken in other ways such as radio telemetry to confirm and sharpen the estimates of the diffusion constant we have obtained in the present analysis. Elsewhere we will present our separate analysis (Abramson et al., 2004) of mice movement data taken from web measurements (Parmenter et al., 2003).

The present analysis has pointed to the importance of the concept of home ranges, which have been known and discussed in many places in the literature earlier (Burt, 1943; Anderson, 1982; Ford, 1979). They would generally be re- 
flected in animal movement measurements in the way we have detailed in our calculations in the present paper and could additionally be of crucial importance in the theory of the spread of epidemics. While they do not appear in the AK description (the tacit assumption in that formalism being that they are larger than other lengths of interest), we have now developed (Kenkre et al., 2004) a series of models to treat them explicitly. The basic idea in our new theory is to consider the dynamics of two types of mice, stationary and itinerant (and susceptible and infected in each category). The stationary mice are the adults that move within their home ranges and do not stray far from the burrow. The itinerant mice are the subadults that must leave to find their own home ranges. Our studies employ a combination of nonlinear analysis and simulations. The results will be presented elsewhere.

\section{Acknowledgements}

It is a pleasure to thank Robert Parmenter for numerous discussions. We also thank Instituto Conmemorativo Gorgas, Drs. Blas Armien and Aníbal Armien, for generously making this work possible. We are grateful to Armando McKency, Mario Ávila, Omar Vargas, Nelson Ríos, Francisco Crespo, Ernest Valdez, Marjorie Hudson, Ricardo Jiménez and Eustiquio Broce for splendid field assistance and other Instituto Gorgas staff for general support. The field work of Gerardo Suzán, Erika Marcé, and Terry Yates was funded by NSF Dissertation Improvement Grant 3-25471-3100. Guillermo Abramson acknowledges partial funding by CONICET (PEI 6482), and by Fundación Antorchas. This work was supported in part by DARPA under grant no. DARPA-N00014-03-1-0900, by NSF/NIH Ecology of Infectious Diseases under grant no. EF-0326757, and by the NSF under grant no. INT-0336343.

\section{References}

Abramson, G., and Kenkre, V. M. (2002). Spatio-temporal patterns in the Hantavirus infection. Physical Review E 66, 011912-1-5.

Abramson, G., Kenkre, V. M., Yates, T., and Parmenter, B. (2003). Traveling waves of infection in the Hantavirus epidemics. Bulletin of Mathematical Biology 65, 519-534.

Abramson, G. (2003). Waves of Hanta, in V. M. Kenkre and K. Lindenberg (Eds.), Modern Challenges in Statistical Mechanics, Patterns, Noise, and the interplay of Nonlinearity and Complexity, , AIP Conference Proceedings volume 658, American Institute of Physics, Melville, NY, USA.

Abramson, G., Giuggioli, L., Kenkre, V. M., Dragoo, J. W., Parmenter, R. R., Parmenter, C. A. and Yates, T. L. (2004). Diffusion and Home Range 
Parameters for Rodents II. Peromyscus maniculatus in New Mexico. Submitted to Ecological Complexity.

Aguirre, M. A., Abramson, G., Bishop A. R. and Kenkre, V. M. (2002). Simulations in the mathematical modeling of the spread of the Hantavirus. Physical Review E 66, 041908-1-5.

Anderson, D. J. (1982). The home range, a new nonparametric estimation technique. Ecology 63(1), 103-112.

Ballard, M., Kenkre, V. M. and Kuperman, M. N. (2004). Periodically varying externally imposed environmental effects on population dynamics. Phys. Rev. E 70, 031912-1-7.

Botten, J., Mirowsky, K., Ye, C., Gottlieb, K., Saavedra, M., Ponce, L. and Hjelle, B. (2002). Shedding and intracage transmission of Sin Nombre hantavirus in the deer mouse (Peromyscus maniculatus) model. Journal of Virology $\mathbf{7 6}(15), 7587-7594$.

Buceta, J., Escudero, C., de la Rubia, F. J. and Lindenberg, K. (2004). Outbreaks of Hantavirus induced by seasonality. Physical Review E 69, 021906$1-8$.

Burt, W. H. (1943). Territoriality and home range concepts as applied to mammals. Journal of Mammalogy 24, 346-352.

Childs, J. E., Ksiazek, T. G., Spiropoulou, C. F., Krebs, J. W., Morzunov, S., Maupin, G. O., Gage, K. L., Rollin, P. E., Sarisky, J., Enscore, R. E., Frey, J. K., Peters, C. J. and Nichol, S. T. (1994). Serologic and genetic identification of Peromyscus maniculatus as the primary rodent reservoir for a new hantavirus in the southwestern United States. Journal of Infectious Diseases 169, 1271-1280.

Ford, R. G. (1979). The analysis of space use patterns. Journal of Theoretical Biology 76, 125-155.

Kenkre, V. M. (2003). Memory formalism, nonlinear techniques, and kinetic equation approaches, in V. M. Kenkre and K. Lindenberg (Eds.), Modern Challenges in Statistical Mechanics, Patterns, Noise, and the interplay of Nonlinearity and Complexity, , AIP Conference Proceedings volume 658, American Institute of Physics, Melville, NY, USA.

Kenkre, V. M. (2004). Results from variants of the Fisher equation in the study of epidemics and bacteria. Physica A 342, 242-248.

Kenkre, V. M., Abramson, G., Giuggioli, L., Neto, G. C. (2004). Generalized Models for the Spread of Hantavirus. University of New Mexico, preprint.

Kuperman, M. N. and Kenkre, V. M. (2004). Spatial features of population dynamics, effects of mutual interactions and of interaction with the environment. Unpublished.

Mills, J. N., Ksiazek, T. G., Peters, C. J. and Childs, J. E. (1999). Longterm studies of hantavirus reservoir populations in the southwestern United States, a synthesis. Emerging Infectious Diseases 5(1), 135-142.

Murray, J. D. (1993). Mathematical Biology, 2nd edition. Springer, New York, USA.

Nichol, S. T., Spiropoulou, C. F.,. Morzunov, S., Rollin, P. E., Ksiazek, T. 
G., Feldmann, H., Sanchez, A., Childs, J., Zaki, S. and Peters, C. J. (1993). Genetic identification of a hantavirus associated with an outbreak of acute respiratory illness. Science 262, 914-197.

Ovaskainen, O. (2004). Habitat-specific movement parameters estimated using mark-recapture data and a diffusion model. Ecology 85, 242-257.

Parmenter, C. A., Yates, T., Parmenter, R. R., Mills, J. N., Childs, J. E., Campbell, M. L., Dunnum, J. L. and Milner, J. (1998). Small mammal survival and trapability in mark-recapture monitoring programs for hantavirus. Journal of Wildlife Diseases 34(1), 1-12.

Parmenter, C. A., Yates, T. L., Parmenter, R. R. and Dunnum, J. L. (1999). Statistical sensitivity for detection of spatial and temporal patterns in rodent population densities. Emerging Infectious Diseases 5, 118-125.

Parmenter, R. R., Yates, T. L. anderson, D. R., Burnham, K. P., Dunnum, J. L., Franklin, A. B., Friggens, M. T., Lubow, B. C., Miller, M., Olson, G. S., Parmenter, C. A., Pollard, J., Rextad, E., Shenk, T. M., Stanley, T. R. and White, G. C. (2003). Small-mammal density estimation, a field comparison of grid-based vs. web-based density estimators. Ecological Monographs $\mathbf{7 3}(1), 1-26$.

Schmaljohn, C. and Hjelle, B. (1997). Hantaviruses, a global disease problem. Emerging Infectious Diseases 3(2), 95-104.

Suzán, G., Marcé, E., Parmenter, R. R., Giermakowski, J. T., Mills, J., Armien, B., Armien, A., Pascale, J. M., Zaldivar, Y., Salazar, J. and T. L. Yates (2004). Responses of hantavirus host communities in Panama to species removal. Presented at the 84th Annual Meeting of the American Society of Mammalogists. 12-16 June 2004. Humboldt State University, Arcata, California, USA.

Vincent, M. J., Quiroz, E., Gracia, F., Sánchez, A. J., Ksiazek, T. G., Kitsutani, P. T., Ruedas, L. A., Tinnin, D. S., Cáceres, L., García, A., Rollin, P., Mills, J., Peters, C. J., and Nichol., S. T. (2000). Hantavirus pulmonary syndrome in Panama, identification of novel hantaviruses and their likely reservoirs. Virology 277, 14-19.

Yates, T. L., Mills, J. N., Parmenter, C. A., Ksiazek, T. G., Parmenter, R. R., Vande Castle, J. R., Calisher, C. H., Nichol, S. T., Abbott, K. D., Young, J. C., Morrison, M. L., Beaty, B. J., Dunnum, J. L., Baker, R. J., SalazarBravo, J. and Peters, C. J. (2002). The ecology and evolutionary history of an emergent disease, Hantavirus Pulmonary Syndrome. Bioscience 52, 989-998. 


\section{A Dependence of $\left\langle x^{2}\right\rangle$ on distribution of initial location of rodents within the grid}

The mean square displacement obviously depends on the initial location of the mice, $x_{0}$. Let us consider the case of infinite home range, but finite $G$, and determine the effect on (11) of the distribution $\mathcal{P}\left(x_{0}\right)$ of initial locations of the mice. We thus evaluate $\left\langle\left(x-x_{0}\right)^{2}\right\rangle$ and calculate its average, denoted by an additional $\langle\cdots\rangle$, with respect to the distribution $\mathcal{P}\left(x_{0}\right)$ of the initial positions through $\left\langle\left\langle\left(x-x_{0}\right)^{2}\right\rangle\right\rangle=\int_{-G / 2}^{G / 2} d x_{0} \mathcal{P}\left(x_{0}\right)\left\langle\left(x-x_{0}\right)^{2}\right\rangle$. Proceeding as in Eq. (11), we have

$$
\begin{aligned}
& \left\langle\left\langle\left(x-x_{0}\right)^{2}\right\rangle\right\rangle= \\
& 2 D t-2 D t \int_{-G / 2}^{G / 2} d x_{0} \mathcal{P}\left(x_{0}\right)\left\{\frac{\left(\frac{G}{2}+x_{0}\right) e^{-\frac{\left(\frac{G}{2}+x_{0}\right)^{2}}{4 D t}}+\left(\frac{G}{2}-x_{0}\right) e^{-\frac{\left(\frac{G}{2}-x_{0}\right)^{2}}{4 D t}}}{\sqrt{\pi D t}\left[\operatorname{erf}\left(\frac{\frac{G}{2}+x_{0}}{\sqrt{4 D t}}\right)+\operatorname{erf}\left(\frac{\frac{G}{2}-x_{0}}{\sqrt{4 D t}}\right)\right]}\right\}+ \\
& \quad+4 \sqrt{\frac{D t}{\pi}} \int_{-G / 2}^{G / 2} d x_{0} \mathcal{P}\left(x_{0}\right) x_{0}\left\{\frac{e^{-\frac{\left(\frac{G}{2}+x_{0}\right.}{4 D}}-e^{-\frac{\left(\frac{G}{2}-x_{0}\right)^{2}}{4 D t}}}{\operatorname{erf}\left(\frac{\frac{G}{2}+x_{0}}{\sqrt{4 D t}}\right)+\operatorname{erf}\left(\frac{\frac{G}{2}-x_{0}}{\sqrt{4 D t}}\right)}\right\} .
\end{aligned}
$$

This reduces to Eq. (11) when $\mathcal{P}\left(x_{0}\right)=\delta\left(x_{0}\right)$. The evolution at short times is found to be independent of $\mathcal{P}\left(x_{0}\right)$ :

$$
\lim _{t \rightarrow 0}\left\langle\left\langle\left(x-x_{0}\right)^{2}\right\rangle\right\rangle \simeq 2 D t
$$

while the saturation turns out to depend on $\mathcal{P}\left(x_{0}\right)$ and is given by

$$
\lim _{t \rightarrow+\infty}\left\langle\left\langle\left(x-x_{0}\right)^{2}\right\rangle\right\rangle=G^{2} / 12+\int_{-G / 2}^{G / 2} d x_{0} \mathcal{P}\left(x_{0}\right) x_{0}^{2} .
$$

Notice that for a uniform initial distribution $\mathcal{P}\left(x_{0}\right)=1 / G$, the integral equals $G^{2} / 12$, and the saturation value is $G^{2} / 6$. This value is twice that obtained for the case of initial placement of the mice at the center of the grid. We see from (A.3) that its right hand side lies always between $G^{2} / 12$ and $G^{2} / 3$ depending on the initial distribution.

The time-dependent evolution of $\left\langle\left\langle\left(x-x_{0}\right)^{2}\right\rangle\right\rangle$ can also be determined when the home range is not infinite. If $x_{0}$ is not the center of the home range, $P(x, t)$ is no longer symmetric with respect to the origin, and the series solution for 
$P(x, t)$ now contains sine (in addition to cosine) functions:

$$
\begin{aligned}
& P_{x_{0}}(x, t)= \\
& \frac{1}{L}+\frac{2}{\alpha \pi} \sum_{n=1}^{+\infty} \frac{\cos \left(\frac{n \pi 2 x_{0}}{L}\right) \sin \left(\frac{n \pi \alpha}{L}\right) \cos \left(\frac{2 n \pi x}{L}\right)}{n} e^{-\frac{(2 n)^{2} \pi^{2} D t}{L^{2}}}+ \\
&+\frac{4}{\alpha \pi} \sum_{n=1}^{+\infty} \frac{\sin \left(\frac{(2 n-1) \pi x_{0}}{L}\right) \sin \left(\frac{(2 n-1) \pi \alpha}{2 L}\right) \sin \left(\frac{(2 n-1) \pi x}{L}\right)}{2 n-1} e^{-\frac{(2 n-1)^{2} \pi^{2} D t}{L^{2}}} .
\end{aligned}
$$

Here the initial probability is

$$
P_{x_{0}}(x, 0)=\left\{\begin{array}{cc}
1 / \alpha & \left|x-x_{0}\right| \leq \alpha / 2 \\
0 & \left|x-x_{0}\right|>\alpha / 2
\end{array} .\right.
$$

We continue to consider the home range and grid window to be concentric for simplicity. The calculation follows the steps shown in earlier cases and yields, for $\left\langle\left\langle\left(x-x_{0}\right)^{2}\right\rangle\right\rangle$, the expression:

$$
\begin{aligned}
& \int_{-G / 2}^{G / 2} d x_{0} \mathcal{P}\left(x_{0}\right) x_{0}^{2}+\frac{G^{2}}{12} \int_{-G / 2}^{G / 2} d x_{0} \mathcal{P}\left(x_{0}\right) \times \\
& \times\left\{\zeta+\frac{6 L}{\zeta^{2} \alpha \pi^{4}} \sum_{n=1}^{+\infty} \cos \left(\frac{n \pi 2 x_{0}}{L}\right) \sin \left(\frac{n \pi \alpha}{L}\right) \times\right. \\
& \times\left[2 \pi \zeta \frac{\cos (n \pi \zeta)}{n^{3}}+\sin (n \pi \zeta)\left(\frac{\pi^{2}}{n^{2}} \zeta^{2}-\frac{2}{n^{4}}\right)\right] e^{-\frac{(2 n)^{2} \pi^{2} D t}{L^{2}}}+ \\
& +\frac{96}{\zeta^{2} \pi^{2}} \frac{x_{0}}{\alpha} \sum_{n=1}^{+\infty} \frac{\sin \left(\frac{(2 n-1) \pi x_{0}}{L}\right) \sin \left(\frac{(2 n-1) \pi \alpha}{2 L}\right)}{(2 n-1)^{2}} \times \\
& \left.\times\left[\zeta \cos \left(\frac{(2 n-1) \pi \zeta}{2}\right)-\frac{2}{\pi} \frac{\sin \left(\frac{(2 n-1) \pi \zeta}{2}\right)}{(2 n-1)}\right] e^{-\frac{(2 n-1)^{2} \pi^{2} D t}{L^{2}}}\right\} \\
& \times\left\{\zeta+\frac{2 L}{\alpha \pi^{2}} \sum_{n=1}^{+\infty} \frac{\cos \left(\frac{n \pi 2 x_{0}}{L}\right) \sin \left(\frac{n \pi \alpha}{L}\right) \sin (n \pi \zeta)}{n^{2}} e^{-\frac{(2 n)^{2} \pi^{2} D t}{L^{2}}}\right\}
\end{aligned}
$$

The time dependence of Eq. (A.6) at short and long times can be shown to tend to that given by Eq. (A.2) and (A.3), respectively, for the case of free diffusion. In the case $L<G$, the result is simply Eq. (A.6) with the parameter $\zeta$ set equal to 1 and $G$ set equal to $L$. In such a case the saturation value is given by an expression similar to Eq. (A.3) but with $L$ replacing $G$ everywhere. 